\title{
Corpus
}

$21 \mid 2020$

Dispositifs numériques et dévoilement de soi

\section{Comment les poly-appelants reconfigurent-ils leurs dires dans leur (re)participation au tchat d'une association qui lutte contre le suicide?}

How do poly-callers reconfigure their statements in their (re) participation in the chat of an association that combats suicide?

Frédéric Pugnière-Saavedra

\section{OpenEdition}

Journals

Édition électronique

URL : http://journals.openedition.org/corpus/5096

DOI : $10.4000 /$ corpus.5096

ISSN : $1765-3126$

Éditeur

Bases; corpus et langage - UMR 6039

Référence électronique

Frédéric Pugnière-Saavedra, «Comment les poly-appelants reconfigurent-ils leurs dires dans leur (re)participation au tchat d'une association qui lutte contre le suicide? », Corpus [En ligne], 21 | 2020, mis en ligne le 09 mars 2020, consulté le 24 janvier 2021. URL : http://journals.openedition.org/ corpus/5096; DOI : https://doi.org/10.4000/corpus.5096

Ce document a été généré automatiquement le 24 janvier 2021.

(c) Tous droits réservés 


\title{
Comment les poly-appelants reconfigurent-ils leurs dires dans leur (re)participation au tchat d'une association qui lutte contre le suicide?
}

\author{
How do poly-callers reconfigure their statements in their (re) participation in \\ the chat of an association that combats suicide?
}

Frédéric Pugnière-Saavedra

1 Cette contribution repose sur une étude d'échanges sous forme de tchats qui émanent d'une association qui lutte contre le suicide ; ces échanges, auto-centrés sur le locuteur qui se dévoile (Kerbrat-Orecchioni et Traverso 2004), constituent en ce sens une pratique discursive particulière dont la finalité pragmatique consiste principalement à être écouté avec empathie et bienveillance par un membre bénévole de l'association. Parler de soi, se livrer, se dévoiler dans un espace numérique particulier (le tchat) amène le locuteur à exposer davantage sa "face négative " via les émotions ressenties du moment (son «territoire » ou ses «réserves» au sens de Goffman) que sa «face positive » (autrement dit « narcissique »).

Dans le cadre de cette étude, nous laisserons volontairement de côté les conversations des primo appelants (les appelants qui n'appellent qu'une seule fois) pour nous centrer sur le contenu des conversations de ceux qui participent plus d'une fois à ce tchat que nous nommons les poly-appelants. Ces derniers n'étant pas identifiés informatiquement ni humainement comme tels dans les échanges ${ }^{1}$, il nous faut procéder à un repérage de marques dans la matérialité langagière des conversations; nous présupposons alors qu'un même scripteur a des pratiques d'écriture stables dans sa morpho-syntaxe (écriture orthografico-phonétique, contraction, apocopes...), la phraséologie, le style (élision par exemple), le niveau de langue... pour extraire un corpus de travail. Nous chercherons alors à identifier les raisons, les besoins qui motivent les appelants à 
(re)participer au forum; Sont-ils insatisfaits du primo-appel ? Le contenu du premier appel est-il incomplet? L'appelant reprend-il la conversation là où il l'avait arrêtée? Quels sont les éléments nouveaux dans le récit des participants? Quel versant du récit est-il mobilisé ? Comment le récit des appelants progresse-t-il ?

\section{Méthodologie pour la constitution du corpus de travail $^{2}$}

3 À partir du corpus de 10 ans d'archive, nous avons dû extraire les conversations qui faisaient l'objet d'une reprise de contact sur le tchat. Nous avons commencé par rapprocher les thèmes, les régularités lexicales et syntaxiques dont certaines renvoient à du discours déjà évoqué avec les amorces de la part de l'appelant telles que « Je vous ai déjà contacté ", "Je me reconnecte " ${ }^{3}$, "J’ai déjà appelé hier ", "Je vous ai parlé il y a quelques jours ", "C'est pas la première fois que je viens sur ce tchat", "Lors de ma participation au tchat du... ", "Je me suis connecté mardi dernier ", "Je vous écris à nouveau ", "J'ai déjà essayé de vous appeler il y a 2 jours» et des éléments structurants sémantiquement «À mon avis je serai vite de retour». Les écoutants ${ }^{4}$ de Chat-Accueil peuvent à leur tour inciter à la reconnexion "et re'chattez" quand vous voulez», "à bientôt alors", "recontactez-nous quand vous en éprouverez le besoin", "revenez vers nous quand vous voudrez »...

4 Les 10 ans d'archives contiennent 18186 conversations qui se répartissent comme suit : 17870 primo-appelants et 316 poly-appelants et sur ces 316 poly-appelants, nous n'avons conservé, grâce à une méthodologie informatique ${ }^{5}$, que 15 conversations qui, après une lecture minutieuse, se sont réduites à 11 pour constituer le corpus de travail (cf. tableau $n^{\circ} 1$ ci-après).

Tableau 1. Extraction des 11 conversations avec le nombre de participations pour constituer le corpus de travail

\begin{tabular}{|c|c|c|c|c|c|c|c|c|}
\hline & Primo appel & Poly appel 1 & Poly appel 2 & Poly appel 3 & Poly appel 4 & Poly appel 5 & Poly appel 6 & Poly appel 7 \\
\hline Conversation 1 & $\begin{array}{c}1381341288 \\
\mathbf{0 9 / 1 0 / 2 0 1 3}\end{array}$ & $\begin{array}{c}1382294276 \\
\mathbf{2 0 / 1 0 / 2 0 1 3}\end{array}$ & & & & & & \\
\hline Conversation 2 & $\begin{array}{c}1318276019 \\
\mathbf{1 0 / 1 0 / 2 0 1 1} \\
\end{array}$ & $\begin{array}{c}1324145436 \\
\mathbf{1 7 / 1 2} / \mathbf{2 0 1 1} \\
\end{array}$ & & & & & & \\
\hline Conversation 3 & $\begin{array}{c}1213818214 \\
\mathbf{1 8} / 06 / 2008\end{array}$ & $\begin{array}{c}1213904116 \\
19 / 06 / 2008\end{array}$ & $\begin{array}{c}1215454009 \\
07 / 07 / 2008\end{array}$ & $\begin{array}{c}1216579742 \\
20 / 07 / 2008\end{array}$ & $\begin{array}{c}1216842270 \\
23 / 07 / 2008\end{array}$ & $\begin{array}{c}1217098275 \\
\mathbf{2 6 / 0 7 / 2 0 0 8}\end{array}$ & $\begin{array}{c}1217699079 \\
\mathbf{0 2} / \mathbf{0 8} / \mathbf{2 0 0 8}\end{array}$ & $\begin{array}{c}1219776481 \\
26 / 08 / 2008\end{array}$ \\
\hline Conversation 4 & $\begin{array}{c}1206821272 \\
\mathbf{2 9 / 0 3 / 2 0 0 8}\end{array}$ & $\begin{array}{c}1206903250 \\
\mathbf{3 0} / \mathbf{0 3} / \mathbf{2 0 0 8}\end{array}$ & $\begin{array}{c}1207680215 \\
\mathbf{0 8} / \mathbf{0 4} / \mathbf{2 0 0 8}\end{array}$ & $\begin{array}{l}1211488487 \\
\mathbf{2 3} / \mathbf{0 5} / \mathbf{2 0 0 8}\end{array}$ & $\begin{array}{c}1211829711 \\
\mathbf{2 6 / 0 5} / \mathbf{2 0 0 8}\end{array}$ & & & \\
\hline Conversation 5 & $\begin{array}{c}1408123639 \\
\mathbf{1 5} / 08 / 2014 \\
\end{array}$ & $\begin{array}{c}1408813225 \\
\mathbf{2 3} / \mathbf{0 8} / \mathbf{2 0 1 4}\end{array}$ & & & & & & \\
\hline Conversation 6 & $\begin{array}{c}1405196263 \\
\mathbf{1 3} / \mathbf{0 7 / 2 0 1 4}\end{array}$ & $\begin{array}{c}1408134404 \\
\mathbf{1 5} / 08 / 2014\end{array}$ & $\begin{array}{c}1408990999 \\
\mathbf{2 5} / \mathbf{0 8} / \mathbf{2 0 1 4}\end{array}$ & & & & & \\
\hline Conversation 7 & $\begin{array}{c}1402421243 \\
\mathbf{1 0} / 06 / 2011\end{array}$ & $\begin{array}{c}1403544910 \\
\mathbf{2 3} / \mathbf{0 6} / \mathbf{2 0 1 4}\end{array}$ & $\begin{array}{c}1403888678 \\
27 / 06 / 2014\end{array}$ & $\begin{array}{c}1404063357 \\
29 / 06 / 2014\end{array}$ & $\begin{array}{c}1404147614 \\
30 / 06 / 2014 \\
\end{array}$ & $\begin{array}{c}1406317628 \\
\mathbf{2 5} / 07 / 2014\end{array}$ & $\begin{array}{c}1422122839 \\
24 / 01 / 2015\end{array}$ & \\
\hline Conversation 8 & $\begin{array}{c}1385839145 \\
\mathbf{3 0} / \mathbf{1 1} / \mathbf{2 0 1 3} \\
\end{array}$ & $\begin{array}{c}1389471952 \\
\mathbf{1 1} / \mathbf{0 1} / \mathbf{2 0 1 4}\end{array}$ & $\begin{array}{c}1391281766 \\
\mathbf{0 1 / 0 2 / 2 0 1 4} \\
\end{array}$ & $\begin{array}{c}1409427322 \\
\mathbf{3 0} / \mathbf{0 8} / \mathbf{2 0 1 4}\end{array}$ & & & & \\
\hline Conversation 9 & $\begin{array}{c}1343584285 \\
29 / 07 / 2012\end{array}$ & $\begin{array}{c}1345396306 \\
19 / 08 / 2012\end{array}$ & $\begin{array}{c}1349723745 \\
\mathbf{0 8} / \mathbf{1 0} / \mathbf{2 0 1 2} \\
\end{array}$ & & & & & \\
\hline Conversation 10 & $\begin{array}{c}1316543035 \\
\mathbf{2 0} / \mathbf{0 9} / \mathbf{2 0 1 1} \\
\end{array}$ & $\begin{array}{c}1316713429 \\
\mathbf{2 2} / \mathbf{0 9} / \mathbf{2 0 1 1}\end{array}$ & $\begin{array}{c}1317061501 \\
\mathbf{2 6} / \mathbf{0 9} / \mathbf{2 0 1 1} \\
\end{array}$ & & & & & \\
\hline Conversation 11 & $\begin{array}{l}1237668699 \\
\mathbf{2 1} / \mathbf{0 3} / \mathbf{2 0 0 9} \\
\end{array}$ & $\begin{array}{c}1238272648 \\
\mathbf{2 8} / 03 / 2009\end{array}$ & & & & & & \\
\hline
\end{tabular}




\section{Cadre théorique}

5 Ces écrits particuliers, qui relèvent de la «communication numérique écrite " (ou «communication écrite en ligne») - (Marcoccia 2016), interpellent fortement la linguistique $\mathrm{du}$ discours tant sur ce qui est dit: comment les participants reconfigurent-ils leur dire lors d'un poly appel que sur le fonctionnement de la ritualisation de la parole, du partage de son émotion, de ses valeurs... Cette production écrite qui vise à énoncer l'objet de l'appel et les raisons du mal-être est avant tout un récit subjectif « il y a des médiations subjectives et culturelles entre l'expérience vécue «brute » et sa mise en récit " [...] où "s'interposent ses schèmes de perception et d'évaluation" (Bertaux, 2010 : 39). Ces schèmes de perception sont «faits de souvenirs, mais aussi de leur mise en perspective, de réflexion continue et d'évaluations rétrospectives. Mémoire, réflexivité, jugement moral, joies et souffrances y contribuent, ainsi que les autres facultés intellectuelles du sujet, ses "équipements" culturels et son idéologie, sa vision du monde et de sa place dans le monde » (Ibid. : 75).

6 Le récit des participants opère donc une reconstruction avec le temps, il reconstruit le passé pour supporter le présent et penser l'avenir. Pour De Gaulejac 2013, « Raconter son histoire est un moyen de "travailler" sa vie » :

- travail sur le passé afin de restaurer, de réparer, de relier, de renouer les fils de la mémoire pour revivifier « le temps perdu».

- travail sur le présent, sur l'histoire « incorporée » c'est-à-dire sur la façon dont elle est agissante en soi aujourd'hui.

- travail sur l'avenir en tant que celui-ci est déterminé par l'histoire. C'est la capacité des hommes de se situer par rapport à leur passé que dépend leur capacité de se projeter ou non dans l'avenir (2013:56).

7 Dans les récits que nous étudions en tant qu'activités «(re)configurant les réalités sociales vécues des locuteurs par le choix des constructions singulières de la syntaxe, du lexique et de formes énonciatives » (Nossik 2011), le participant commence souvent par la fin, à savoir par ce qui empêche de bien vivre, par l'évocation des entraves qu'il tente d'expliquer ou de justifier. Nous appréhenderons la mise en mots de ces entraves sous le prisme de l'analyse de discours (tendance française) qui prend appui sur les marques indicielles et qui considère qu'il existe des invariants, des propriétés spécifiques linguistiques et discursives pour rendre observables des configurations spécifiques de ce type de récits.

8 La méthodologie d'analyse de discours sera complétée par deux approches: une approche dialogique (Bakthine 1978 et Bakthine et Volochinov 1977, Bres et Mellet 2009) et une approche textométrique qui articule synthèses quantitatives et analyses à même le texte (Lebart \& Salem 1994).

9 Mayaffre (2007:9) précise : « Encore moins que la fréquence d'un mot, la récurrence de segments ne peut être naïvement attribuée au hasard : soit elle pointe une contrainte syntaxique, soit elle indique une détermination ou option sémantique », il poursuit en 2012 en affirmant que la textométrie permet :

- «d'analyser un grand nombre de documents textuels que ni l'œil ni la mémoire ne sont capables de prendre en compte dans une telle marée de mots ;

- de mettre en perspective les caractéristiques qui ne se révèlent que par rapport à un ensemble plus vaste ; 
- d'atteindre la précision, la systématicité, l'exhaustivité ou l'objectivité nécessaire pour une description scientifique ;

- d'ouvrir des voies de l'interprétation historique loin de celles induites par l'actualité contemporaine, nos a priori, nos sujets favoris puisque l'outil ne ressent lui ni préférence, ni aversion » (2012:14-16).

\section{Analyse de corpus}

10 À partir des 11 conversations retenues que nous aborderons transversalement, nous nous centrerons ensuite longitudinalement sur les deux conversations faisant l'objet du plus grand nombre d'appels. Des moments de "réglage » sur le fonctionnement du tchat, sur ce que peuvent (vs ne peuvent pas) dire les écoutants en fonction des règles déontologiques fixées par l'association, sur les attentes des appelants, apparaissent très tôt dans l'échange. Émergent ainsi des éléments dans la (in)constance dans la pratique d'écriture des appelants (section 3.1), puis à travers un pacte tacite de coopération (section 3.2) apparait une double contrainte d'écriture (section 3.3), une construction en écho à partir de la procédure de thématisation (section 3.4) et une construction fortement dialogique des énoncés (section 3.5).

\subsection{Des pratiques d'écriture inconstantes dans l'échantillon retenu}

11 Avant d'aborder les régularités linguistiques proprement dites (section 3.2 et subséquente), nous avons voulu identifier des régularités constitutives dans la temporalité des pratiques d'écriture. Nous avons voulu savoir combien de jours séparaient le primo appel des poly appels (cf. tableau $\mathrm{n}^{\circ} 2$ ) mais le trop petit nombre de conversations ne permet pas de dégager des tendances significatives.

Tableau 2. Nombre de jours écoulés entre chaque version ${ }^{6}$

\begin{tabular}{|c|c|c|c|c|c|c|c|}
\hline & $\begin{array}{c}\text { nb de jours entre } \\
\text { PAet PA1 }\end{array}$ & $\left|\begin{array}{c|}\text { nb de jours } \\
\text { entre PA1 et PA2 }\end{array}\right|$ & $\begin{array}{l}\text { nb de jours entre } \\
\text { PA2 et PA3 }\end{array}$ & \begin{tabular}{|c|} 
nb de jours entre PA3 \\
et PA4
\end{tabular} & \begin{tabular}{|c|} 
nb de jours entre \\
PA4 et PA5
\end{tabular} \mid & $\begin{array}{c}\text { nb de jours entre } \\
\text { PA5 et PAG }\end{array}$ & $\begin{array}{l}\text { nb de jours } \\
\text { entre PAG et } \\
\text { PA7 }\end{array}$ \\
\hline Conversation 1 & 11 & & & & & & \\
\hline Conversation 2 & 68 & & & & & & \\
\hline Conversation 3 & 1 & 18 & 13 & 3 & 3 & 7 & 24 \\
\hline Conversation 4 & 1 & 9 & 45 & 3 & & & \\
\hline Conversation 5 & 7 & & & & & & \\
\hline Conversation 6 & 33 & 10 & & & & & \\
\hline Conversation 7 & 13 & 4 & 2 & 1 & 25 & 183 & \\
\hline Conversation 8 & 42 & 21 & 210 & & & & \\
\hline Conversation 9 & 21 & 50 & & & & & \\
\hline Conversation 10 & 2 & 4 & & & & & \\
\hline Conversation 11 & 7 & & & & & & \\
\hline
\end{tabular}

Nous émettions également l'hypothèse que le nombre de formes décroit au fil des versions; le primo appel consistant à présenter les raisons du mal-être, les versions 
subséquentes des poly appels seraient expurgées de cette mise à nu et favoriseraient l'expression du mal-être dans un autre versant. Il n'en est rien. Si nous examinons les deux conversations avec le plus grand nombre de poly appels (fig. 1 et 2 portant sur les conversations 3 et 7), nous constatons qu'il est difficile de dégager encore une fois une tendance car l'écoutant pouvant être différent, l'appelant est alors dans l'obligation de reformuler son dire et des évènements personnels peuvent intervenir, nécessitant une réorganisation du récit. Figure 1. Nombre de formes par version
pour l'instance $n^{\circ} 3$

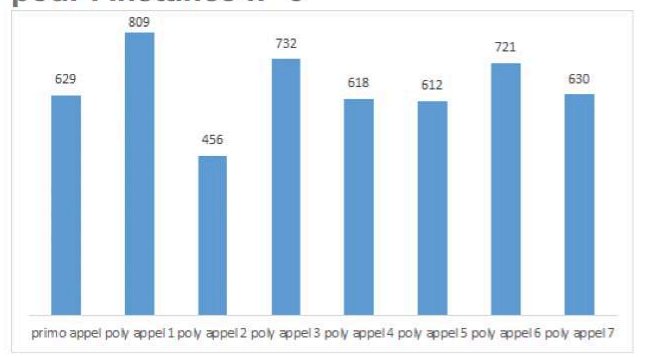

Figure 2. Nombre de formes par version pour l'instance $n^{\circ} 7$

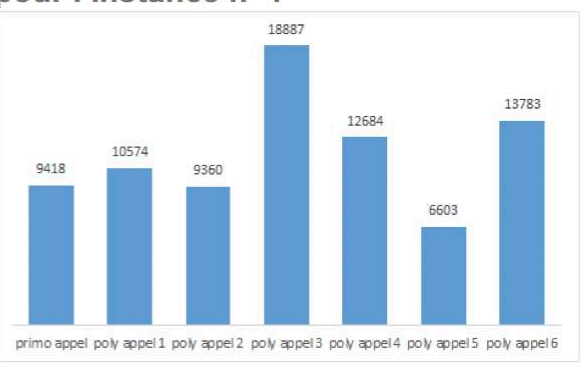

\subsection{Un dispositif énonciatif particulier qu'écoutants et appelants doivent accepter et s'approprier}

Cette association lutte contre le suicide en écoutant les appelants qui expriment leur détresse, leur mal-être. L'écoute est confidentielle, anonyme ${ }^{7}$, hors de tout jugement et dans de respect de la personne; les écoutants constituent une équipe, la parole de l'appelant est libre.

(Session : 1206903250)

Chat-Accueil (21:01:53): 14 ans, oui pour répondre à votre interrogation, il n'y a pas d'âge pour être sur ce "chat". Il est là pour vous, pour dire, écrire ce qui ne va pas, ce que vous ne pouvez dire facilement à quelqu'un d'autre.

Un des objectifs de l'écoute consiste en une introspection pour faire « avancer un peu » l'appelant.

(Session : 1206903250)

Chat-Accueil (21:28:15): tu as raison, il [le web tchat] ne va pas permettre de revenir en arrière, mais ne peut-il pas te permettre d'avancer un peu?

Sans que cela ne soit explicitement dit, les écoutants peuvent consacrer entre 40 minutes et une heure à chaque appelant, au-delà ces derniers s'orientent vers la fin de l'interaction en initiant la phase de clôture de l'échange

(Session : 1211488487)

Chat-Accueil (00:16:31): je vous souhaite succès dans votre relation

Chat-Accueil (00:16:41): Au plaisir d'une prochaine rencontre

Chat-Accueil (00:16:48): et surtout merci aussi pour la confiance

Appelant (00:17:17): merci, moi aussi j'aimerais bien rediscuter avec vous un jour;

bonne nuit

Chat-Accueil (00:17:26): bonne nuit

Votre correspondant a quitté cette session. (10:09:46 pm)

(Session : 1213818214)

Chat-Accueil(21:39:12): En tous cas moi j'ai confiance en toi, malgré les bêtises. Je vais te laisser car nous parlons depuis longtemps mais tu sais que tu peux nous joindre aussi souvent que tu le souhaites, tu tomberas sur d'autres personnes qui, comme moi, essaieront de t'aider. J'espère avoir réussi à le faire 
mais je n'en suis pas sure, en tous cas je te remercie de m'avoir accordé ta confiance et j'espère que notre conversation se poursuivra avec un énorme câlin avec tes parents, et cette nuit j'enverrai l'étoile du Berger, celle qui brille le plus, veiller sur toi

Appelant (21:41:29): vous êtes super gentils. oui je ferai un câlin à papa et maman, promis. et aussi c'était bien de parler avec vous. papa il a dit que si je veux, on va se promener tous les 2, que nous 2, sans mes cousins. Merci vous êtes super sympa Chat-Accueil (21:42:59): oui rien que vous deux, c'est une belle idée. Merci Gaël. Appelant (21:43:09): bonne nuit

Appelant (21:43:11): merci

Chat-Accueil (21:44:04): au revoir, bonne très bonne nuit

Votre correspondant a quitté cette session.(09:44:10 pm)

ou en prétextant que d'autres appelants en détresse sont dans l'attente d'un échange.

(Session : 1207680215)

Chat-Accueil (23:03:48): je vais devoir prendre d'autres personnes dans quelques instants

Chat-Accueil (23:04:42): peut-être devriez-vous réfléchir à ce lien à vos parents, à votre place maintenant dans la famille

Appelant (23:05:44): d'accodac et aussi je vais dire à tonton pour la pocket bike. alors merci et je vais réfléchir merci parce que vous êtes super sympa, comme on imagine Sos amitié

Chat-Accueil (23:06:13): merci

Chat-Accueil (23:06:16): bonne soirée

Appelant (23:06:27): bonne nuit

Chat-Accueil (23:06:45): pareillement

Votre correspondant a quitté cette session. (11:06:46 pm)

(Session : 1405196263)

Chat-Accueil (00:01:33): Vous avez une psy (dans 1 mois) qui, même si vous la critiquez, semble vous aider au mieux; et puis il y a, de temps en temps SoS Amitié qui ce soir va hélas être obligé d'arrêter car il y a d'autres intervenants qui doivent occuper ce site... Relisez tout ce que nous avons échangé, il y a tout de même de la matière. Vous pouvez nous contacter aussi par téléphone

L'appelant, quant à lui, privilégie ce canal car il permet de raconter des fragments de vie à quelqu'un qu'il ne connait pas

(Session : 1211488487)

Appelant (20:56:29): [...] et sur un forum, c'est cool de parler avec quelqu'un qu'on ne connait pas

Il permet également de s'exprimer de manière très discrète (pour les adolescents notamment)

(Session : 1349723798)

Appelant (22:23:17): ouais , merci , papa et maman ils pensent que je tchate avec des copains alors ils sont contents mais j'ai conscience de leur mentir.

Pour la génération des milléniaux, l'écran est le dispositif plébiscité pour ce forum car moins chargé que la voix (pas de genre, de timbre, de mélodie, d'intonation, de silences fortement expressifs ou d'émotion).

(Session : 1318276019)

Chat-Accueil (23:29:18): je ne peux que vous proposer d'utiliser le téléphone et la messagerie de SOS Amitié qui sont aussi des moyens mis à votre disposition pour exprimer votre douleur.

Chat-Accueil (23:29:50): mais un peu différents et qui peuvent peut-être vous aider Appelant (23:30:17): j’ai déjà essayé

Appelant (23:30:53): une fois je n'ai pas réussi à parler et la seconde fois je suis tombée sur un homme 
Appelant (23:30:53): j'ai raccroché

Appelant (23:30:53): d'ailleurs vous êtes peut-être un homme?

Appelant (23:30:59): mais par écran interposé c'est différent, moins effrayant

(session : 1317577573)

Appelant (20:30:33): parler oralement ne me soulage pas, je suis plus à l'aise à en parler avec vous derrière un écran que face à ma psy

Appelant(20:30:55): la honte est trop présente et les mots ne sortent pas toujours

(Session 1257883796)

Appelant (21:31:34): il me semble que j'ai beaucoup plus de facilités à m'exprimer par le biais de messagerie comme celle-ci par exemple que par tel notamment, (Marcoccia, 2000) mais il permet surtout de favoriser l'exposition des tranches de vie expurgées de trace d'émotion, ce que ne permet pas la voix.

(Session : 1349723745)

Chat-Accueil (21:49:58): oui, vous pouvez téléphoner à Sos amitié...il y a 49 numéros en France

Appelant (21:50:03): non, je veux pas, j’ai pas le courage de prendre le téléphone, ça me demande beaucoup d'effort... j'ai pas envie de me retrouver à pleurer au bout de la ligne, je sais c'est nul ms bon..

Chat-Accueil (21:50:35): c'est comme vous le ressentez bien sur

21 Les appelants reformulent leurs dires à chaque connexion dès lors que les écoutants ne sont pas les mêmes et qu'ils ne sont pas tous exposés aux mêmes couvertures média

(Session : 1213818214)

Appelant (21:00:07): et elle a dit que l'accident ça fait longtemps mais, c'est vrai, parce que c'était le 2 juin

Chat-Accueil (21:01:15): un accident le 2 juin ? il faudrait m'expliquer parce qu'ici, on se relaie et on ne connaît pas tout ce qui s'est échangé

Appelant (21:02:06): ben, j'étais dans l'accident de Allinges vous savez ce qu'il y a eu?

Chat-Accueil (21:02:14): non, qu'est-ce qu'il y a eu ?

Appelant (21:03:41): http://tf1.lci.fr/infos/france/faits-divers/0,3866268,00collision-allinges-drame-questions-.html

Appelant (21:05:05): ben on est a eu un accident avec mes copains et un train nous a dégomé et Tom est mort et aussi Yannis et Tim et Léa et Fanny et Benoit et Natacha et

Mais les appelants manifestent explicitement leur surprise à l'idée de tomber sur le même écoutant qui assure la permanence :

Session : 1211488487

Chat-Accueil (22:36:03): je croyais que vs aviez déconnecté parce que ma question était un peu tarte

Appelant (22:36:33): génial, j'ai de la chance comme ça je ne dois pas tout recommencer. Non, je ne fais pas ça et je n'ai pas eu votre question

De même, ils expriment dans de nombreux cas et explicitement leur mécontentement à l'idée d'avoir à reformuler leur pensée à chaque nouvelle connexion en cas de nouvel écoutant.

(Session : 1211488487)

Chat-Accueil (20:58:01): oui.. aimeriez-vous nous en dire +

Appelant (20:58:17): ce qui est chiant c'est que je l'ai raconté tout à l'heure mais

comme ça a coupé alors voilà

Appelant (20:58:40): j'ai 14 ans au fait alors j'ai le droit 
Chat-Accueil (20:58:53): oui, dsl... toutefois la collègue ma rapidement évoquer... Il vous est possible de poursuivre. son histoire dans ses axes les plus saillants si l'écoutant est différent ; et celle consistant à faire progresser son récit en mentionnant des éléments narratifs qui justifient l'appel si l'écoutant est le même. Dans les deux cas, l'appelant évoque les éléments de sa vie en (re)configurant sa pensée, c'est ce qu'illustrent les faits saillants dans les Analyses Factorielles des Correspondances (AFC) ${ }^{8}$ des conversations 9, 3 et 7 (voir figures cidessous). Ces représentations graphiques montrent qu'un même participant mobilise différemment les distributions lexicales disponibles pour évoquer sa situation lors des différentes connections. Pour la conversation $n^{\circ} 9$, on constate toutefois une proximité (relative) entre les versions 1 et 3 des poly-appels.

Figure 3. AFC pour la conversation 9

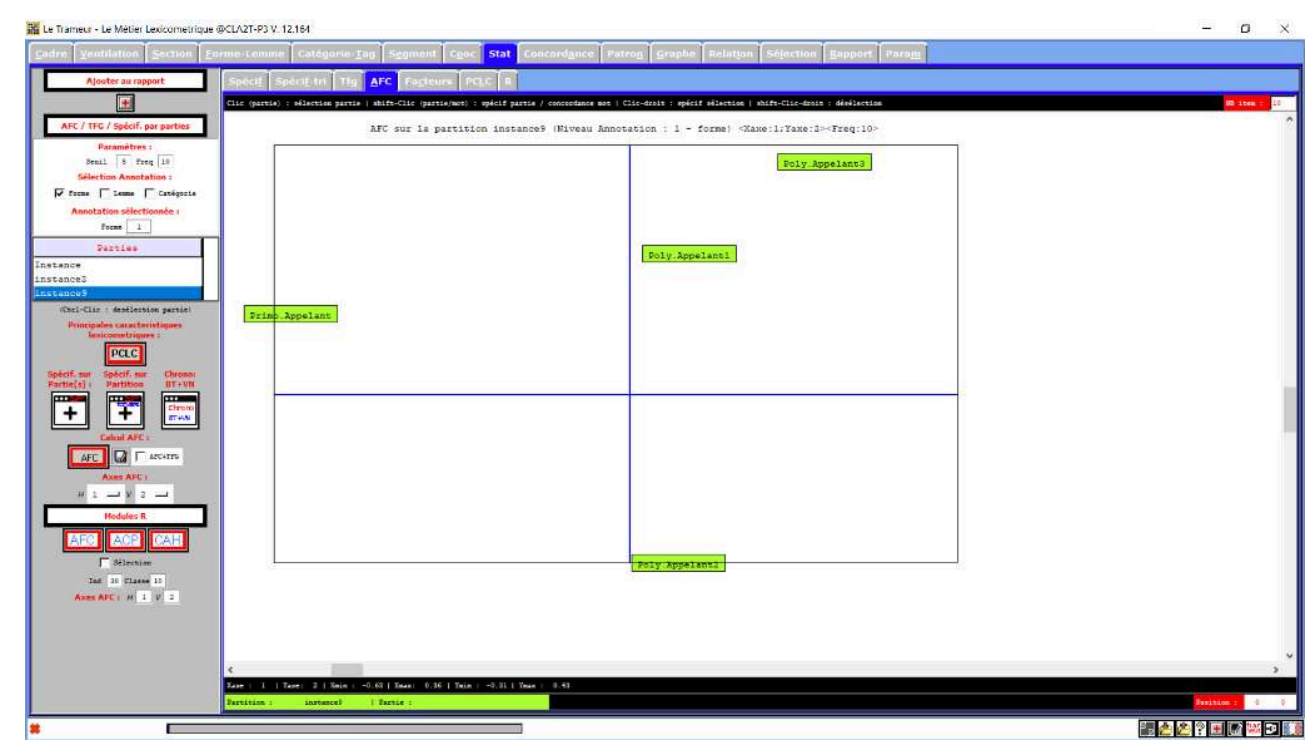

Nous porterons notre intérêt sur les échanges des deux conversations ( $n^{\circ} 3$ et 7$)$ qui ont le plus de versions mais qui fonctionnent sur des modes discursifs différents : pour l'une, les marques dialogales et dialogiques (cf. infra 3.5) sont très présentes et pour l'autre, elles sont absentes, le récit mobilise alors d'autres stratégies de discours.

La conversation $\mathrm{n}^{\circ} 3$ se compose de 8 versions, et d'après le graphe ci-après, on peut formuler l'hypothèse que 4 regroupements ${ }^{9}$ constituent chacun une unité sémantique avec des éléments d'une grande proximité lexicale et syntaxique, c'est une hypothèse d'autant valide que les échanges suivent la chronologie de l'histoire. 
Figure 4. AFC pour la conversation $n^{\circ} 3$

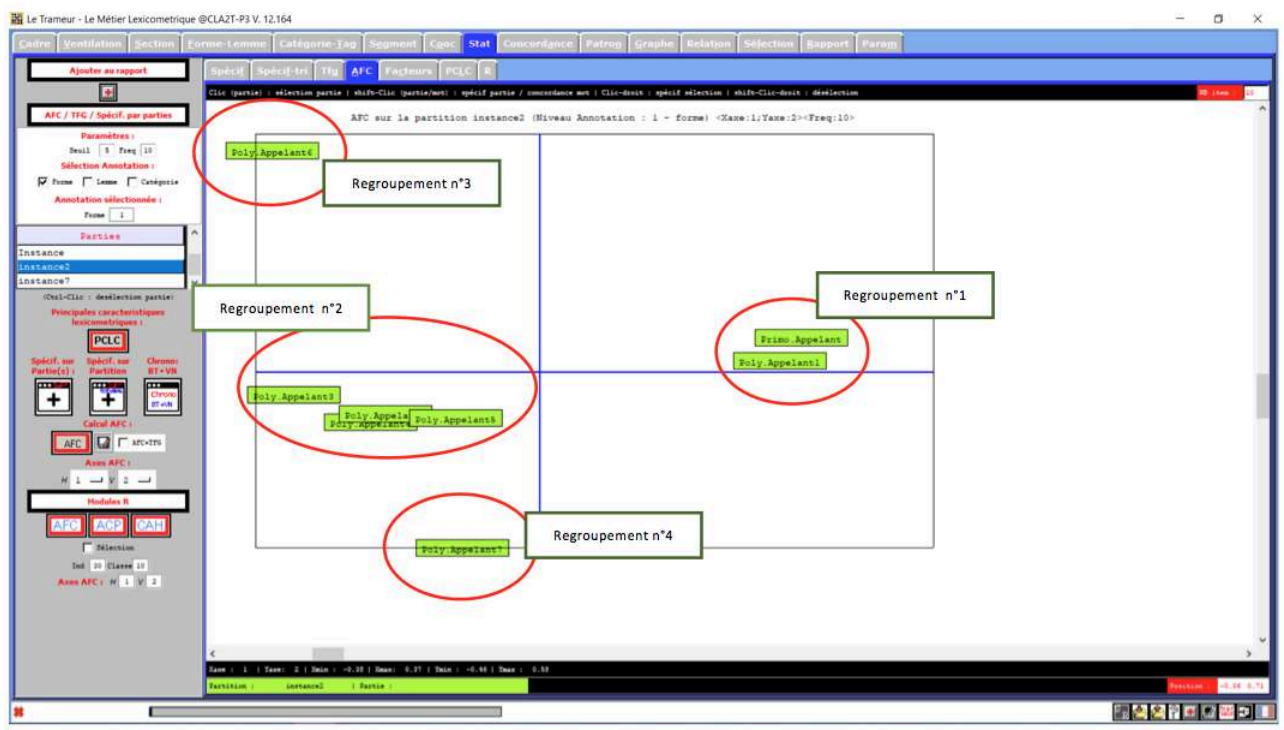

Les échanges de cette instance portent sur l'émotion d'un adolescent en classe de cinquième qui évoque un accident de car qui a eu lieu le 2 juin 2008 avec 25 blessés et 7 morts lors d'un voyage scolaire de fin d'année à Allinges. Le car est resté bloqué à un passage à niveau et un train l'a percuté.

Appelant (22:06:07): ben parce que y a des choses que j'arrive à écrire comme sur le forum et sur votre site. J'ai vu sur le forum que vous existez mais je veux pas le dire avec ma bouche

Appelant (22:08:37): moi je dormais à moitié dans le bus en écoutant la musique et ensuite Quentin il a dit "on est bloqué, le train va nous foncer dedans" et Tom a gueulé "merde voilà un train et pendant ce temps monsieur François il gueulait sur le chauffeur "bougez votre bus" et le chauffeur il y arrivait pas alors monsieur François il a crié tout «le monde à l'avant » mais moi je ne pensais pas que ça allait être grave

Appelant (22:12:02): ensuite y a eu des cris tout le monde se levait pour aller vers l'avant et le train il nous a dégomés et comme ma tête a cogné fort je me souviens plus trop et je ne sais pas qui m'a aidé à sortir, c'est un prof et dehors j'ai vu qu'il y avait des morts, j'ai vu Léa, une basket de Tom et j'avais du verre dans la bouche, on saignait tous à cause du verre. Je l'ai dit à mon papi aujourd'hui, je lui ai dit tout ce que je viens de vous écrire quand on est allé à la pêche mais si je dis ça à maman elle va pleurer je crois

Appelant (22:13:59): et papa je ne lui ai pas dit parce que

Chat-Accueil (22:14:02): oui, peut-être qu'elle pleurerait mais je me demande si cela ne la rassurerait pas un peu de voir que vous pouvez sortir avec des mots toute votre souffrance

Une entrée par les spécificités par partie montre que le regroupement 1 relate les circonstances de l'accident de car et les difficultés du collégien à mettre en mots ce qu'il ressent avec ses parents; le regroupement 2 évoquent la manière dont l'enfant ressent le besoin de se reconnecter pendant la période de congés et fait part à l'écoutant des bêtises qu'il a commises pendant l'été; le regroupement 3 évoque le chagrin qu'il ressent et le regroupement 4 évoque son refus de suivre un soutien psy.

\section{Contenu sémantique de chaque regroupement}

31 - Regroupement 1: le collégien relate les circonstances de l'accident de car et ses difficultés à mettre en mots ce qu'il a vécu, son émotion et ce qu'il ressent avec ses 
parents qui sont eux-mêmes enseignants et amis du Principal du collège où les enfants étaient scolarisés. Une exploration du corpus par les spécificités supérieures à 10 des substantifs fait émerger les lexèmes maman, papa, bus, accident, pleure, et illustrent ces éléments de contextualisation qui justifient l'appel. Une autre exploration par les verbes à l'infinitif montre que, outre les verbes polysémiques être (25 occurrences), dire (22 occurrences) faire ( 18 occurrences) et voir ( 15 occurrences), le premier semi auxiliaire le plus utilisé est retourner (9 occurrences) construit avec le modal vouloir dans sa forme négative : « je ne veux pas retourner au collège ».

Figure 5. Concordancier de retourner

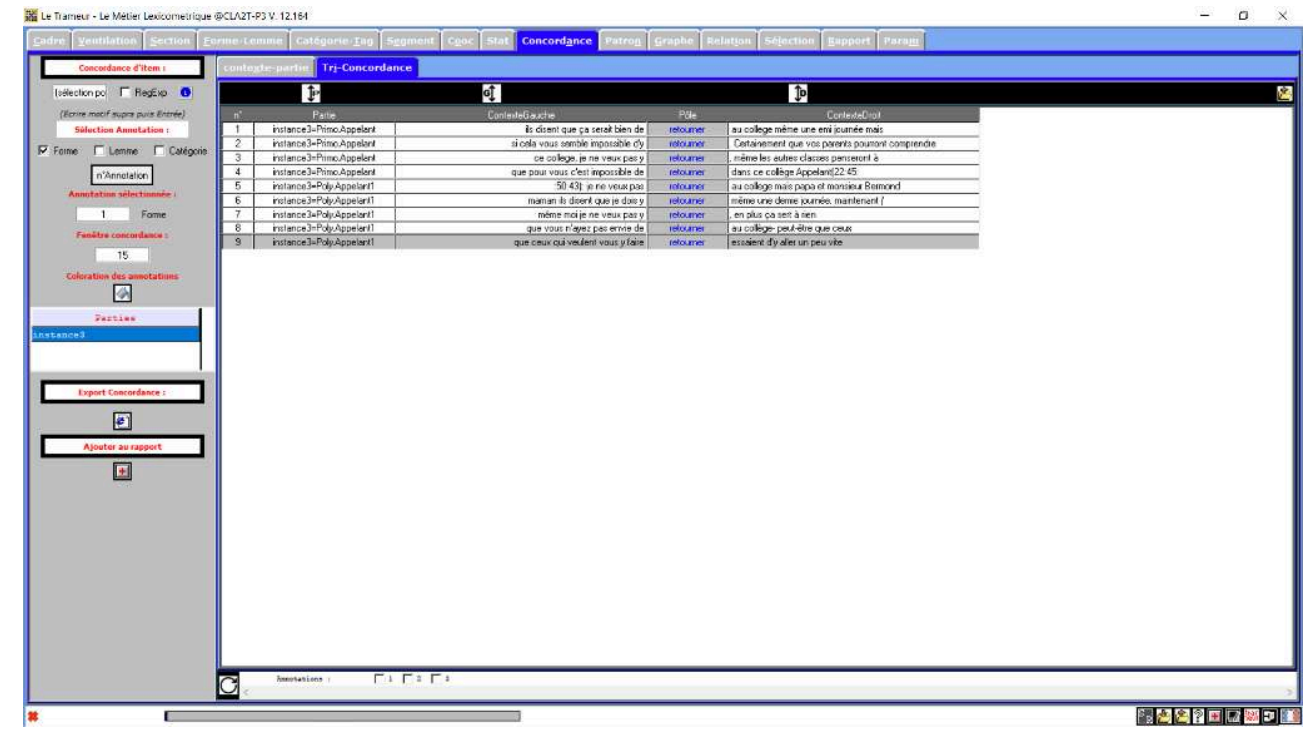

Il en est de même pour les formes verbales conjuguées : on trouve par ordre décroissant d'apparition les verbes polysémiques ai (119 occurrences), est (82 occurrences), dit (31 occurrences) et en $4^{\mathrm{e}}$ place, veux ${ }^{10}$ ( 27 occurrences) très majoritairement utilisé dans sa forme négative ( $67 \%$ des cas) et dans une moindre mesure dans une construction infinitive exprimant le désiratif ( $29.5 \%$ des cas).

Tableau 3. Construction du segment veux

\begin{tabular}{|c|c|c|}
\hline & $\begin{array}{c}n b \\
\text { occurrences }\end{array}$ & $\%$ \\
\hline formes négatives (soit avec les 2 morphèmes ne ...pas ou uniquement pas) & 18 & 67 \\
\hline construction infinitive exprimant le désiratif (faire des calins, changer de collège partir, fuir, retirer mon platre) & 8 & 29,5 \\
\hline construction complétive (je veux aussi que mon visage...) & 1 & 3,5 \\
\hline total & 27 & 100 \\
\hline
\end{tabular}

- Regroupement 2 : les obsèques ayant eu lieu à la fin du mois de juin, les échanges portent à la fois sur la relation adolescent et parents et sur la manière dont le collégien redoute les vacances. Les spécificités supérieures à 10 portant sur les relations familiales (papa [indice de spécificité 28], maman [indice de spécificité 23], parents [indice de spécificité 18], cousin [indice de spécificité 13]), sur les conditions de l'accident (nuit [indice de spécificité 14], accidents [indice de spécificité 14]) et sur la manière dont se déroulent les vacances [indice de spécificité 14] et les bêtises [indice de spécificité 10] qu'il commet avec son cousin dans un village vacances

Appelant (21:30:47): j'ai des copains ici [village vacance] mais le gardien il est venu parce qu'on a fait des bêtises et aussi avant hier, j'étais avec Gaétan, un gars qui est en vacances ici et papa il m'a vu en train de fumer et de boire une bière et il 
est venu, je l'avais pas vu, il a pris la cigarette, il l'a écrasée et il a pris la bière et il l'a vidé et il a dit "pas de ça Gael" et il a dit à Gaétan toi non plus. On était caché derrière le resto, mais il a fait tout ça sans s'énerver mais il me piste, il me surveille tout le temps, ça me saoule.

Chat-Accueil (21:31:36): c'est son rôle tu le sais bien..

Appelant (21:31:43): non, mes cousins ils deviennent des crétins, ils n'osent rien faire, ils écoutent tout et tout le temps

Chat-Accueil (21:32:41): fumer et boire de la bière, c'est montrer que tu es grand? c'est faire des bêtises pour embêter les adultes? je pense aussi que la bière peut te saouler plus que ton père...

Appelant (21:34:13): non, parce que j'ai envie c'est tout. On a pris la voiture électrique du village vacances et on a fait des tours la nuit, j'étais sorti en cachette mais les vigils nous ont attrapés et ils m'ont ramené au chalet

Appelant (21:35:08): pas avec mes cousins hein, eux ils ont peur de tout Chat-Accueil (21:35:23): tu savais que tu prenais ce risque-là...c'est comme si tu voulais te mettre en danger pour conjurer la peur

Appelant (21:36:19): ben ils ont galéré pour nous attraper, j'ai pas eu peur Chat-Accueil (21:37:04): tu n'as pas peur ou bien tu essaies de voir jusqu'où tu n'auras pas peur??

Chat-Accueil(21:38:19): tu n'as donc pas peur de provoquer les vigiles et tu fais semblant de dormir qd ton père vient vers toi par peur de lui parler... c'est bizarre, tu ne trouves pas, $\mathrm{Tu}$ sembles vouloir qu'on ne te saoulent plus avec tout ce drame, qu'on ne te regarde plus et, d'un autre côté tu fais un peu tout pour te faire remarquer...

Les poly-appels de l'adolescent montrent qu'il a besoin de montrer qu'il fait des bêtises pour attirer l'attention de ses parents

Appelant (21:57:38): mais maintenant papa et maman ne se fâchent pas comme avant

Appelant (21:58:15): je ne sais pas, le prof de jet-ski ne veut plus que je vienne parce que je ne l'ai pas écouté et je suis parti trop loin, il est devenu fou

Chat-Accueil (21:59:30): décidément, tu les cherches!! ça veut dire quoi "ils ne se fâchent plus comme avant"? quelle est la différence à tes yeux?

Appelant (21:59:41): mais je suis dégouté, je ne pourrai pas faire de jet ski, mes cousins ils les veut bien encore, ben je fais ce que je veux, ils se fâchent un peu c'est tout, ils m'ont pas puni

Chat-Accueil (22:02:37): Le prof de jet-ski ne peut effectivement plus t'accepter si tu n'as pas respecté les règles de sécurité. Ne cherches-tu pas alors à provoquer tes parents pour qu'ils réagissent comme avant? Ils n'osent plus te punir pensant peut-être que tu as vécu qqchose de dur alors que tu souhaiterais que tout soit comme avant?

Chat-Accueil (22:03:26): y compris leur attitude vis à vis de toi. Pourquoi ne leur distu pas tout cela?

Comme dans le regroupement 1 , la négation est omniprésente mais s'est décentrée, elle ne porte plus sur le fait de ne plus vouloir " retourner au collège » ou de «changer de collège » mais sur le fait de ne plus "vouloir pleurer", de ne plus vouloir que "ses parents se fâchent » à cause de son attitude. 
Figure 6. Répartition de ne dans le regroupement 2

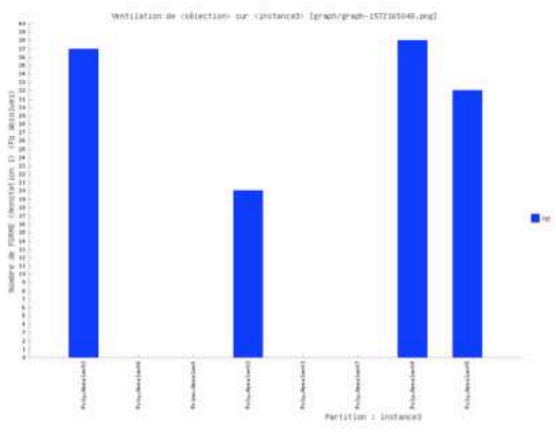

Tableau 4. Lemme verbal sur lequel porte la négation en nombre

d'occurrences

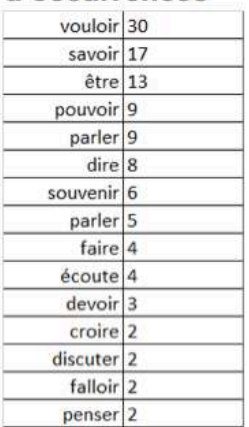

Tableau 5. Répartition des connecteurs pour le regroupement 2

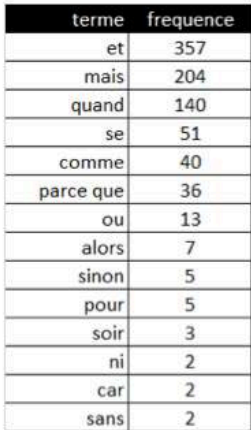
tableau $n^{\circ}$ 5) qui valorise ce qui est post-posé (désir de cesser d'être méchant, désir d'être trop soutenu dans ce moment difficile) et qui disqualifie l'état de méchanceté, l'état de se sentir bien dans la famille.

Appelant (20:36:42): je suis trop méchant avec papa et maman mais pourtant je ne veux pas mais je le fais quand même

je suis trop nul parce que je veux rester qu'avec eux depuis l'accident mais des fois en même temps ils m'énervent d'être très proches de moi

ils comprennent, ils ont dit qu'ils savent que je suis très en colère mais que c'est mieux d'en parler que de s'énerver comme ça entre nous.

- Regroupement 3 : l'appelant exprime ses difficultés à conjurer les moments où il fait des bêtises et consulte encore régulièrement le Blog de Tom (enfant décédé dans l'accident). L'écoutant le fait réfléchir pour qu'il exprime « autrement » ses souffrances et les angoisses consécutives à l'accident et l'invite notamment à écrire.

- Regroupement 4 : l'appelant fait part de son refus de coopération avec un psy qui, à ses yeux, ne lui apporte rien.

Appelant (20:49:21): je suis Gael et j'ai fait l'effort de ne pas venir plusieurs jours mais aujourd'hui j'ai un truc à dire

Appelant (20:49:35): j'ai été chez le psy et c'était encore nul

Appelant (20:49:38): psy

Chat-Accueil (20:49:47): le psy est nul ?

$[\ldots]$

Appelant (21:10:06): je m'appelle Gael et depuis juin, je viens souvent

Chat-Accueil (21:11:05): c'est ce que vous m'avez dit. Je crois comprendre que les visites chez le psy, c'est lié à l'accident

Appelant (21:12:38): oui papa et maman ils veulent mais moi j'ai pas envie, j'en ai vu plein au collège et à la rentrée y en aura encore plein, surtout parce qu'on est pas retourné en classe depuis l'accident, enfin, les blessés et aussi pour monsieur Jandin [enseignant qui accompagnait la classe et qui s'est suicidé pendant les vacances]

Chat-Accueil (21:14:13): c'est probablement ça la difficulté : ce n'est pas vous qui avez décidé mais papa et maman. C'est parfois pas facile à avaler. En même temps, je comprends que l'on propose un accompagnement à quelqu'un qui a vécu un accident de ce genre, si dramatique

Appelant (21:14:59): mais même à l'enterrement y en avait plein des psys

Chat-Accueil (21:15:45): oui, probablement pour bien faire mais ce serait important que vous décidiez vous-même comment vous voulez continuer, en parler à papa et 
maman peut être ? Est-ce possible?

Appelant (21:16:23): je ne veux pas de psy mais papa et maman ils veulent parce qu'en plus j'ai fait plein de conneries en vacances de cette instance portent sur une appelante qui exprime son mal-être en allant au travail car, incomprise de son chef, elle se sent harcelée par ses collègues.

Figure 7. AFC pour conversation $\mathrm{n}^{\circ} 7$

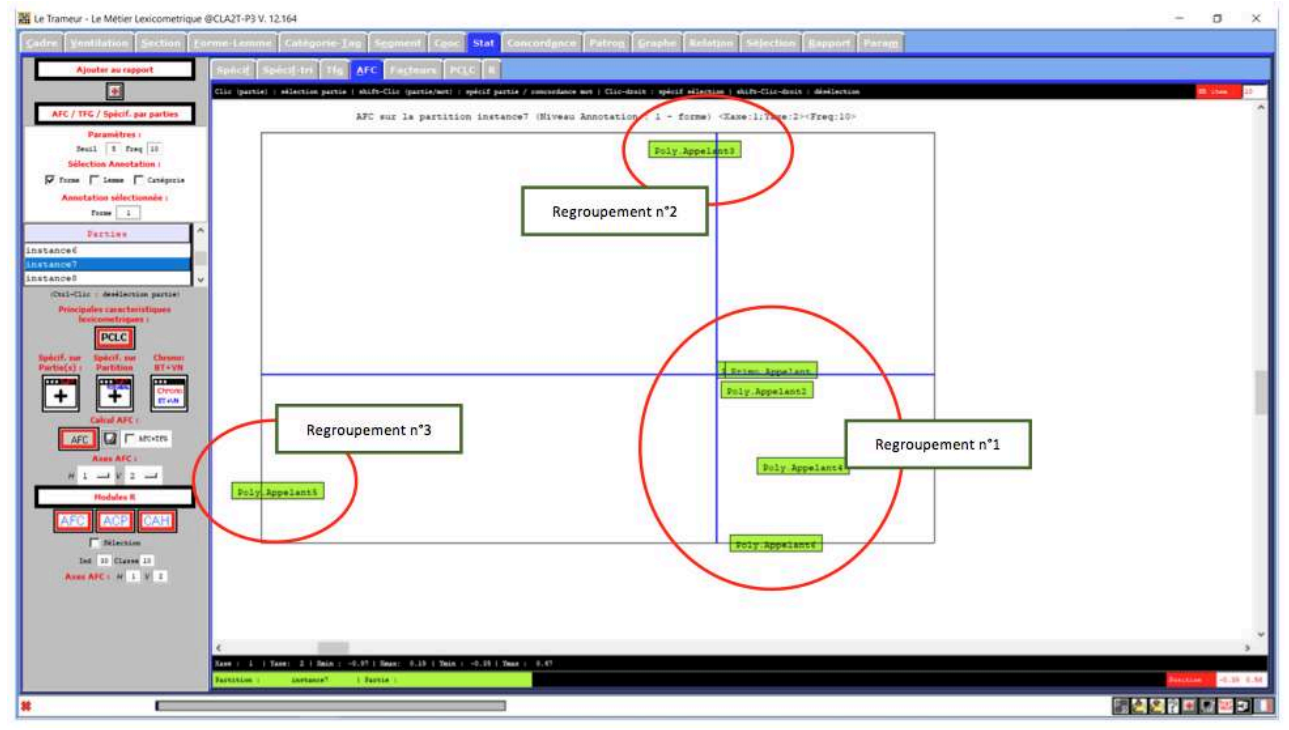

41 Contenu sémantique de chaque regroupement

- Regroupement 1 : dans les 5 versions qui composent ce regroupement, l'appelante fait part de son malaise et de ses difficultés au travail. Ses collègues féminines sont méchantes, l'appelante se sent harcelée et son chef ne prend pas de décision. Une exploration par les spécificités par ordre décroissant fait ressortir une forte présence des propos portant sur son chef [indice de spécificité 46], sur le travail [indice de spécificité 23], sur ses collègues [indice de spécificité 19] et sur la manière dont elle parle 
de sa situation et sur la recherche d'arguments pour montrer à son patron qu'elle est une bonne salariée : preuve [indice de spécificité 15], comportement [indice de spécificité 12], argument [indice de spécificité 12], problème [indice de spécificité 10].

Si l'on explore les poly-appels qui constituent ce regroupement, on constate que le terme «chef » est très largement cooccurrent (cf. tableau $\left.n^{\circ} 4\right)$ du pronom personnel sujet « elles » qui renvoie aux collègues femmes de travail et de la recherche d'éléments (de preuves) qui montrent que l'appelante se sent victime d'injustice car elle risque de perdre son emploi.

Tableau 4. Cooccurrences de Chef

\begin{tabular}{|l|l|r|}
\hline Pôle & Cooccurrent & FQ(cooc) \\
\hline $\begin{array}{l}\text { chef } \\
\text { chef }\end{array}$ & elles & 255 \\
\hline chef & preùgues & 26 \\
\hline chef & poste & 18 \\
\hline chef & contrat & 14 \\
\hline chef & réunion & 12 \\
\hline chef & méchante & 11 \\
\hline chef & entreprise & 11 \\
\hline chef & méchantes & 10 \\
\hline chef & malhonnetes & 9 \\
\hline chef & rh & 6 \\
\hline chef & renouvelé & 6 \\
\hline chef & démotivée & 3 \\
\hline & & 3 \\
\hline
\end{tabular}

Tableau 5. Constructions syntaxiques de

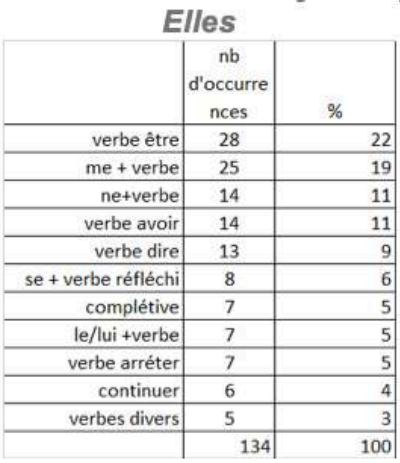

Une exploration par la manière dont l'appelante parle de ses collègues féminines (cf. fig. $n^{\circ} 5$ ci-dessus) montre qu'elle utilise une construction attributive avec une axiologie négative (avec parfois un intensifieur) dans une majorité des cas ( $22 \%$ des cas) : elles sont: méprisantes, malhonnêtes, subjectives, (tellement) butées, malveillantes, affreusement méchantes, (vraiment) folles, très (trop) sures d'elles, dans leur tort, de (très) mauvaise foi.

Il est également intéressant de lister les verbes (dans $19 \%$ des cas) dont l'appelante pense faire l'objet: Elles me haïsent, détestent, me démolissent, me traitent avec mépris, m'ignorent, font comme si j'existais pas, me provoquent, me font passer pour une femme méchante, me culpabilisent, me pourrissent la vie, me dégoutent, m'écoeurent, m'empoisonnent, m'accusent injustement

- Regroupement 2, composé du poly-appel 3, porte sur la réunion de service en tant qu'objet de discorde. Dès que l'appelante prend la parole en réunion, elle a l'impression que ses collègues la dévalorisent et dès que ses collègues prennent la parole, l'appelante a l'impression d'être ignorée. L'appelante rédige des listes qui reportent les remarques désagréables de ses collègues mais le chef ne souhaite manifestement pas prendre parti.

- Regroupement 3, composé du poly-appel 5, porte sur la perception réciproque de manque de respect de l'Autre.

Du point de vue du contenu sémantique des poly appels des conversations 3 et 7 , on constate qu'on aborde, à chaque connexion, le problème sous un angle complémentaire qui donne une cohérence au tout mais sans jamais être tout à fait satisfait car l'écoutant peut certes écouter avec empathie mais ne peut complètement résoudre le problème de mal-être de l'appelant.

Nous aborderons dans la section suivante, la mise en mots des motifs des poly-appels au sein d'une même instance. 


\subsection{Construction en écho de la thématisation de la raison de l'appel}

\section{la raison de la (re)connexion. On s'oriente vers la spécification de la nature de ce qui ne} vapas.

51 L'appelante recourt à une première désignation " problème » qui en soi contient peu d'ancrage référentiel car c'est en discours qu'il s'actualise; en l'occurrence ici un " problème dans le travail ", le mot problème étant ici en lien avec une résolution venue de l'extérieur. Dans le poly-appel $n^{\circ} 1$, le motif apparait sous le lexème "difficulté » actualisé avec l'adjectif « grave », avec un ancrage plus centré sur la personne «j'ai une difficulté qui est grave ».

Vient ensuite (poly-appel $n^{\circ}$ 2) la nature de la difficulté: le harcèlement dans son travail ; puis dans le poly-appel $n^{\circ} 3$ la nature du harcèlement est évoquée dans son versant paroxystique «je n'arrive plus à m'en sortir ». Le poly-appel $n^{\circ} 4$ se construit avec une reprise de la première désignation actualisée « $\mathrm{j}$ 'ai de graves problèmes de travail» et le poly-appel $n^{\circ} 5$ évoque une spécification de cet état «harcèlement moral » avec les personnes qui sont à l'origine du harcèlement au travail, le poly-appel $\mathrm{n}^{\circ} 6$ évoque, quant à lui, une des raisons de cet état de mal-être: son incapacité à convaincre son chef de la malhonnêteté de ses collègues.

L'étude de ces amorces montre au moins deux choses :

- l'idée selon laquelle l'appelant reprend son histoire là où il l'a laissée lors d'un appel antérieur ne se manifeste pas ici puisqu'à chaque fois, les motifs de l'appel sont reprécisés dans une paradigme assez stable.

- le spécification de ce qui ne va mobilise un paradigme stable mais qui ne va pas totalement crescendo avec deux registres de dénomination qui apparaissent : problèmes, difficultés dans le travail (primo et poly appel 1 et 4 ), le harcèlement dans les relations humaines dans l'entreprise (poly appels 2, 3, 5 et 6).

\subsection{Hétérogénéité des formes dialogiques pour alimenter la textualité des poly-appels.}

54 Tout énoncé se produit toujours en interaction avec d'autres énoncés, il est, à ce titre, doublement dialogique (Bakthine 1978 et Bakthine et Volochinov 1977) dans la mesure 
où il entretient avec l'énoncé deux types de relations: une relation interlocutive (dialogue externe), et une relation interdiscursive (dialogue interne). Plus tard, Bres $(2005,2006,2007,2008,2011)$ qui a consacré une grande partie de ses recherches à la notion de Dialogisme, ajoute aux deux dimensions interlocutive et interdiscursive, celle d'intralocutive (dialogue avec soi), (cf. Bres 2017).

La situation de discours des poly appels dans ce tchat implique une forte attitude réflexive de l'appelant (situation de dialogisme intralocutif) qui en même temps qu'il met en mots sont mal être, réfléchit à sa propre condition, à la manière dont il tape son message à la fois dans la forme et dans le fond, à la manière dont ce qu'il dit peut être perçu par l'écoutant dont il a une représentation. La dimension intralocutive des échanges initiés par l'appelant favorise par conséquent des constructions interdiscursives qui les lient à l'écoutant et qui font partie de leur textualité ${ }^{12}$ au sens de Détrie, Siblot, Verine (2001 : 349).

\subsubsection{Du discours de reformulation à l'emprunt}

Dans la perspective rogérienne, l'écoutant incite l'appelant à reformuler son dire en changeant de catégorie grammaticale (passage du verbe au substantif par exemple) avec une emphase et l'usage des guillemets.

Appelant (19:45:28): elles sont toujours très contentes de me mépriser et avoir monté le chef contre moi leur donne de l'assurance

Chat-Accueil (19:47:07): Comment se manifeste le "mépris" de votre chef? Cela peut-il vous faire craindre pour votre emploi?

57 L'écoutant, par des formes de "discours en train de se faire" peut renvoyer à un discours autre, à du discours d'emprunt (Authier-Revuz, 2001 : 192), recourt à des guillemets impliquant une forme de distance et de perplexité de sa part.

Appelant (19:31:37): par exemple, elles me traitent toujours avec mépris, puis lorsque je leur parle, elles disent que ce ne sont que des impressions de ma part.

Chat-Accueil (19:33:17): que veulent-elles dire par des "impressions" de votre part?

Dans les exemples suivants, l'emploi des termes «efficace » et « harcèlement " par l'écoutant est emprunté à l'appelant avec emphase et reformulation de ce mot en tant que mot clé de la séquence.

Appelant (20:06:48): cependant, je dois être efficace car à la longue, si cette affaire dure, il sera énervé et ne voudra, comme vous dites, plus être convaincu, mais chercher à se débarrasser du problème.

Chat-Accueil (20:12:56): Vous êtes très lucide ! Le mot "efficace" est le bon.

Appelant (19:48:56): à chaque fois qu'il y a une rencontre avec elles, j'ai une sorte de représailles de sa part

Appelant (19:49:37): J'aimerais trouver les mots pour le convaincre car elles me harcèlent vraiment

Chat-Accueil (19:51:18): Si vous avez peur pour votre emploi, peut-être serait-il utile de vous renseigner sur le plan juridique, car le "harcèlement" peut être puni par la loi.

Dans l'exemple suivant, la mise à distance avec les guillemets autour du substantif amie met l'accent sur une amie qui, non seulement ne l'est plus mais encore, qui s'est transformée en rivale car elle a séduit son petit ami.

Appelant (21:39:50): j'ai retrouvé récemment sur Facebook certaines personnes perdus de vue

Appelant (21:40:08): dont Morgane, une "amie" que je connaissais du lycée vite fait 
60

présence des guillemets dans ce type d'énoncés ne se limite pas à reprendre un terme d'autrui, ils amorcent une mise à distance en mettant la focale sur un élément qui participe à discréditer un comportement ou à dévaloriser une personne.

\subsubsection{Du dialogisme interlocutif au dialogisme interdiscursif}

61

Le dialogisme interlocutif s'oriente vers le tour de parole antérieur de l'allocutaire dans les genres dialogaux et vers la réponse de l'allocutaire qu'il sollicite et sur laquelle il anticipe la réponse.

On perçoit dans l'appel suivant le fait que la question «Vous avez déjà parlé avec moi »? destiné à un même écoutant (ou à un autre) va reconditionner le dire de l'appelant.

Chat-Accueil (19:45:04): bonsoir

Appelant (19:45:37): bonsoir, je suis déjà venu plusieurs fois, je suis Gael, j'étais dans l'accident de bus à Allinges, vous avez déjà parlé avec moi ?

Appelant (19:48:34): oui

Chat-Accueil (19:50:55): Bonsoir Gaël vous êtes venu plusieurs fois mais je n'ai jamais communiqué avec vous. Vous étiez dans l'accident du bus, et sans doute avez déjà parlé de ce terrible accident qui nous a tous bouleversés, si vous le souhaitez nous pouvons parler tous les deux sans vous sentir obligé de me raconter depuis le début, car je ne voudrais pas risquer de vous faire souffrir en réactivant des souvenirs douloureux

Appelant (19:51:38): non de toute façon, je ne voulais pas le raconter encore une fois

Les marques d'interlocution succèdent à l'expression d'une préférence parmi les écoutants bénévoles.

Appelant (20:53:50): vous avez vu, monsieur Martin il s'est suicidé, j'ai lu sur le net, c'était notre prof d'histoire, il nous a accompagné pendant le voyage Chat-Accueil (20:54:15): oui, je l'ai appris et j'ai pensé très fort à toi Appelant (20:55:08): c'est vrai ? vous être super sympa et même si j'ai discuté avec 3 personnes et ben je sais le soir ou c'était avec vous parce que vous êtes la plus sympa

Dans la séquence suivante, le marqueur concessif «Quand même» renvoie à une séquence d'ouverture portant sur le souvenir et sur des conséquences induites.

Appelant (22:01:58): mais aussi des fois depuis l'accident, je fais pipi au lit

Appelant (22:02:13): mais papa et maman ils ont dit que c'est pas grave mais j'ai l'impression que ça m'a quand même choqué

Dans l'énoncé suivant, l'appelant reprend un fragment de discours prononcé "juste » par le psy qui n'a pas la même valeur pour chacun d'eux. Pour le psy, le problème relationnel est minoré alors que pour l'appelant, il s'agit d'un problème d'une réelle importance.

Appelant (19:44:23): c'est "juste" un problème relationnel m'a dit le psy! Vous imaginez?

Dans l'énoncé suivant, l'écoutant pose une question pour ouvrir sur une séquence où l'appelant est amené à évoquer ce qui lui plaisait

Chat-Accueil (20:19:41): À part la voiture électrique et le saut dans les rochers, qu'est-ce que tu aimes... ou tu aimais avant 
Appelant (20:19:54): le skate

Appelant (20:20:03): et papa et maman et mes copains et l'ordi plateforme, il ne poursuit pas son récit à l'endroit où il l'a laissé avec un écoutant précédent, il reformule son dire à destination d'un autre écoutant, ce qui revient à dire que l'appelant ne livre jamais tout à fait le même récit bien qu'il s'agisse d'une même histoire de vie. montrent que les appelants sont en attente d'une écoute attentive ; une écoute qui est le résultat de l'histoire singulière d'une adulte sans sa situation professionnelle actuelle (conversation $\mathrm{n}^{\circ} 7$ )

Chat-Accueil (20:02:39): je vous sens totalement obnubilée par cette affaire et je le conçois bien sûr...mais il vous faut prendre de la hauteur pour ne pas vous détruire...prendre conseil de qqn de l'exterieur?

Appelant (20:02:41): il y a des témoins, mais ils ne veulent pas parler, vous savez, j'ai juste besoin de quelqu'un qui me donne un peu de soutien moral et je vous remercie de m'écouter

Ou une écoute qui peut contenir une forme de déculpabilité collective pour le jeune collégien qui a vécu un véritable drame lors de son voyage d'école de fin d'année. Ce drame collectif ayant été fortement médiatisé, on peut penser qu'il y a un double interdiscours qui alimente la conversation : celui de la couverture médiatique qui peut intervenir sur l'information de l'écoutant et sur son empathie à l'encontre de cet accident d'enfants et celui de ses camarades rescapés qui peuvent alimenter une forme de culpabilité 


\section{BIBLIOGRAPHIE}

Authier-Revuz J. (2001). « La représentation du discours autre : un champ multiplement hétérogène ", in J.-M. Lopez-Munoz, S. Marnette, L. Rosier (éd.), Le discours rapporté dans tous ses états : question de frontières, actes du colloque Bruxelles 8-11 novembre 2001. Paris : L'Harmattan, 35-53.

Bakhtine M. (1978) [1934]. « Du discours romanesque », in Esthétique et théorie du roman. Paris : Gallimard, 83-233.

Bakhtine M. (1984) [1952]. « Les genres du discours », in Esthétique de la création verbale. Paris : Gallimard, 265-308.

Bertaux D. (2010). Le récit de vie, Armand Colin, collection 128 "L'enquête et ses méthodes », $3^{e}$ édition, Paris.

Bres J. (2017). «Dialogisme, éléments pour l'analyse », Recherches en didactique des langues et des cultures [En ligne], 14-2, mis en ligne le 15 juin 2017, consulté le 17 septembre 2019. URL : http://journals.openedition.org/rdlc/1842; DOI : 10.4000/rdlc.1842.

Bres J. et Mellet S. (éd.) (2009). « Dialogisme et marqueurs grammaticaux », Langue Française 163.

De Gaulejac V. (2013). «Produire une histoire et chercher à en devenir le sujet : pour une clinique de l'historicité ", in C. Niewiadomski \& C. Delory Momberger (dir.), La mise en récit de soi. Paris, Éditions Téraèdre, 53-64.

Détrie C. Siblot P. \& Verine B. (2001). Termes et concepts pour l'analyse du discours. Une approche praxématique.

GAUDUCHEAU N. « La communication des émotions dans les échanges médiatisés par ordinateur : bilan et perspectives ", Bulletin de psychologie, 2008/4 (Numéro 496), 389-404. DOI : 10.3917/bupsy.496.0389. URL : https://www.cairn.info/revue-bulletin-de-psychologie-2008-4page-389.htm.

Kerbrat-Orecchioni C. et Traverso V. (2007). Confidence, dévoilement de soi dans l'interaction. Niemeyer.

Lebart L. \& Salem A. (1994). Statistique textuelle. Paris : Broché.

Marcoccia M. (2000). « Les smileys : une représentation iconique des émotions dans la communication médiatisée par ordinateur ». Les émotions dans les interactions communicatives, 249-263.

Marcoccia M. (2016). Analyser la communication numérique écrite, Paris, A. Colin, coll. Icom. Série Discours et communication, $160 \mathrm{p}$.

Mayaffre D. (2012). Nicolas Sarkozy. Mesure et démesure du discours Presses de Sciences Po, Références.

Niewiadomski C \& Delory-Momberger C. (2013). La mise en récit de soi. Presses universitaires du Septentrion.

Nossik S. (2011). «Les récits de vie comme corpus sociolinguistique : une approche discursive et interactionnelle », Corpus 10,119-135.

Todorov T. (1981). Mikhail Bakhtine. Le principe dialogique, suivi de Écrits du cercle de Bakhtine. Paris : Minuit. 


\section{ANNEXES}

\section{Annexes 1 : Méthodologie en 5 phases pour construire le corpus de travail}

Phase 1 : étiquetage des néo et des poly-appelants en favorisant la précision :

Si le mot « déjà » a été rencontré dans les deux premiers tours de parole, l'appelant est considéré comme revenant produisant ainsi un poly-appel.

Si le mot « déjà » n'a pas été rencontré dans toute la conversation, l'appelant est considéré comme "nouveau », on garde alors le même nombre de nouveaux que de revenants pour construire et équilibrer le modèle d'apprentissage

Phase 2 : construction d'un modèle d'apprentissage grâce à l'utilisation de l'algorithme de descente de gradient stochastique (SGD) à partir d'un étiquetage (316 revenants, 316 nouveaux).

Phase 3 : interprétation du modèle d'apprentissage obtenu (plus le score est élevé, plus le terme est discriminant dans le modèle d'apprentissage) :

Tableau : discrimination des termes

\begin{tabular}{|c|c|c|c|c|c|}
\hline \multicolumn{2}{|c|}{$\begin{array}{c}\text { a) Termes les plus } \\
\text { discriminants avec les Uni- } \\
\text { grammes }\end{array}$} & \multicolumn{2}{|c|}{$\begin{array}{c}\text { b) Termes les plus } \\
\text { discriminant des revenants : }\end{array}$} & \multicolumn{2}{|c|}{$\begin{array}{l}\text { c) Termes les plus discriminant } \\
\text { des nouveaux : }\end{array}$} \\
\hline déjà & 2.485572 & déjà & 1.227432 & bonjour & 0.587591 \\
\hline plus & 0.013115 & si & 0.195933 & bonsoir & 0.475105 \\
\hline fait & 0.010859 & fait & 0.185745 & sais & 0.383577 \\
\hline fois & 0.009721 & fois & 0.120814 & merci & 0.327569 \\
\hline bonsoir & 0.008848 & bien & 0.058427 & tout & 0.185371 \\
\hline ans & 0.008566 & ans & 0.057155 & plus & 0.158612 \\
\hline si & 0.008541 & depuis & 0.046966 & parler & 0.158145 \\
\hline merci & 0.008527 & tout & 0.022460 & ans & 0.138750 \\
\hline bien & 0.008517 & parler & 0.015773 & depuis & 0.120755 \\
\hline bonjour & 0.008410 & plus & -0.001418 & bien & 0.120444 \\
\hline
\end{tabular}

Phase 4 : application du modèle afin d'étiqueter les 18186 conversations, ces extractions aboutissent au constat suivant : 17870 primo-appelants et 316 poly-appelants. Nous n'avons pas pris en compte les conversations courtes (moins de 20 tours de parole). Nous avons dû ensuite déterminer quel était le premier appel à partir duquel il $\mathrm{y}$ a eu une deuxième, une troisième participation au tchat... cette recherche informatique a fait ressortir 15 échanges de poly-appelants (sur 316) qui se répartissent comme suit :

Tableau : Extraction informatique de corpus 
Image 10005E940000399A00002772576FCCCFD8EF4C09.emf

\begin{tabular}{|c|c|c|}
\hline Conversation & Primo appe lant ( $n^{\circ}$ Session) & Poly appelant ( $n^{\circ}$ Session) \\
\hline 1 & 1235507521 & 1235508555 \\
\hline 2 & 1381341288 & 1382294276 \\
\hline 3 & 1318276019 & 1317577573 \\
\hline 4 & & $\begin{array}{l}1217098275,1216842270,1217699079,1213904116, \\
1216579742,1219776481,1213818214,1215454009\end{array}$ \\
\hline 5 & & $\begin{array}{c}1207680215,1206821272,1206903250,1211829711, \\
1211488487\end{array}$ \\
\hline 6 & 1408813225 & 1408123639,1408813225 \\
\hline 7 & 1405196263 & 1408134404,1408990999 \\
\hline 8 & $\begin{array}{l}1402421243,1406317628, \\
140388.8678,1422122839, \\
1403544910,1404063357\end{array}$ & 1404147614 \\
\hline 9 & 1409427322 & $1391281766,1389471952,1385839145$ \\
\hline 10 & 1345396306 & 1349723745,1343584285 \\
\hline 11 & 1324145436 & 1319303334 \\
\hline 12 & 1318276019 & 1317577573 \\
\hline 13 & 1316543035 & 1316713429,1317061501 \\
\hline 14 & 1257883796 & 1257713311 \\
\hline 15 & 1237668699 & 1238272648 \\
\hline
\end{tabular}

Phase 5 : recherche de parenté entre nouveau (primo-appels) et revenant (poly-appels) grâce à l'utilisation de n-grammes de caractères afin de modéliser les conversations en caractérisant principalement les proximités lexicales qui permettent de rapprocher les mêmes personnes.

Une première étape consiste à regarder pour chaque poly-appelant, quels sont les primoappelants les plus proches afin d'avoir une liste de primo-appelants dont on sait qu'ils vont revenir.

Une seconde étape consiste, pour chaque primo-appelants identifié, à regarder les polyappelants les plus proches qui sont extraits à la suite d'une vérification manuelle pour repérer les incohérences en termes de genre (repérage des accords masculin $v s$ féminin), d'âge (style, contractions éventuelles, apocopes, niveau de langue) ou de date de conversation (la date d'une re-participation au tchat ne peut être antérieure à celle de la participation pour la première fois) pour vérifier du point de vue du contenu que la conversation du primo appelant identifiée informatiquement correspond bien à celle.s. du poly-appelant.

La lecture attentive des 15 conversations a permis d'en éliminer $\mathbf{4}$ qui n'étaient pas cohérentes. Le corpus de travail s'élève donc à 11 conversations.

\section{Annexes 2. Répartition du segment veux}

Figure 7. répartition du segment veux 


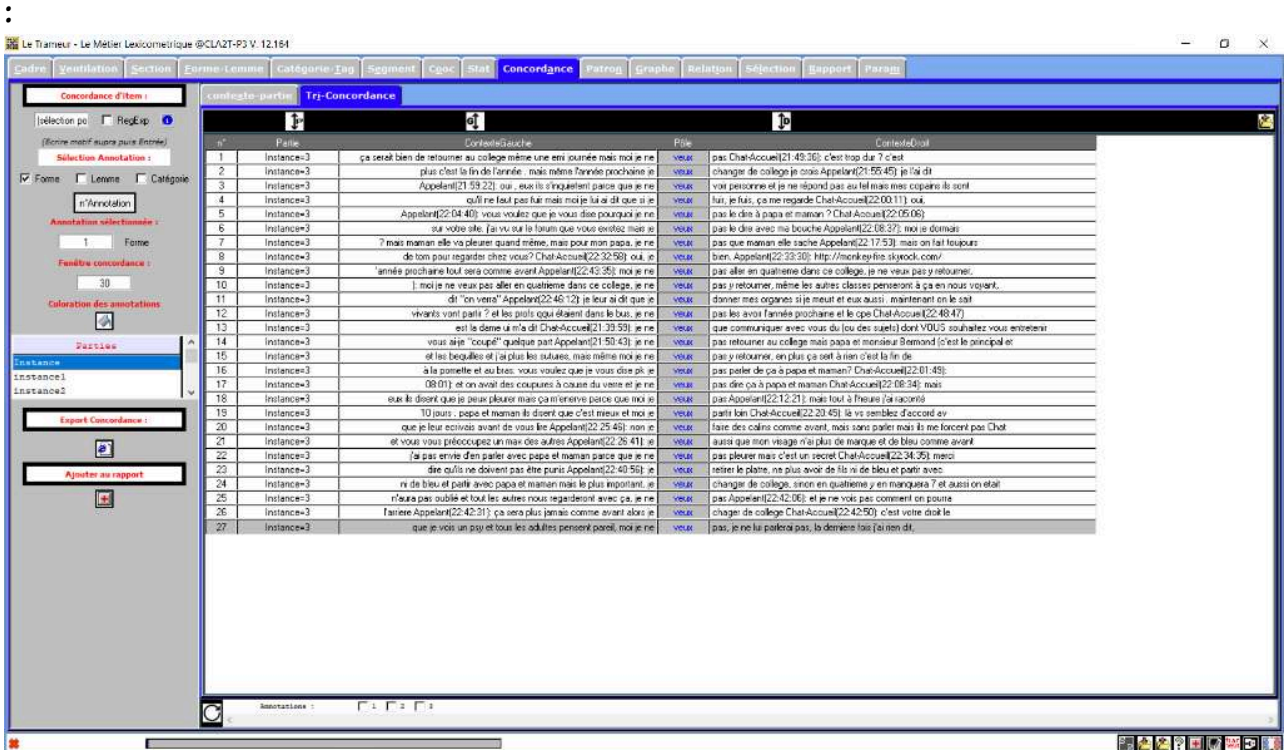

\section{NOTES}

1. En effet, chaque connexion génère automatiquement l'attribution d'un numéro de session sans prendre en compte le nombre d'appel(s) pour un même appelant.

2. Je remercie Nicolas Bechet, MCF en informatique au laboratoire IRISA, UMR CNRS 6074 de l'Université Bretagne Sud pour la fouille de données préalable à la constitution du corpus.

3. De même que les lemmes construits à partir de « reconnecter».

4. Voir ici même la contribution de Ledegen \& Wagner sur le «nous » de surformalité largement utilisé par les écoutants.

5. Cf. en annexe 1 : la méthodologie informatique en 5 phases utilisées pour isoler le corpus de travail.

6. Dans le tableau, PA signifie Primo Appelant, PA1, Poly Appelant $n^{\circ} 1$, PA2, Poly Appelant $n^{\circ} 2$ etc.

7. L'anonymat va jusqu'à contourner les nombreuses demandes des appelants qui consistent à savoir si l'écoutant est un homme ou une femme, détail important pour la construction de leur récit et la compréhension de la situation par l'écoutant.

Appelant (20:55:45): Non ça me va très bien! Vous êtes une femme ou un Homme ? (juste ça et promis je pose plus rien comme question) c'est pour savoir si je peux demander des conseils en fonction du "sexe" de la personne, ça peut aider aussi!

Chat-Accueil (20:56:34): non cela n'aide pas car nous sommes tous honnêtes et droits dans nos bottes

Appelant (20:57:56): Je parle de par exemple, si vous êtes un homme vous pourrez me donner de conseils par rapport à mon ex ( vu que vous savez comment réagisse les hommes) si vous êtes une femme vous me comprendrez très bien par rapport au fait que mon amie se rapproche de mon ex et de la tristesse que je peux ressentir

Chat-Accueil (20:58:21): et aussi, vous pouvez constater que nous donnons quelques indications pas des conseils, nous explorons avec vous vos pistes (comme celle d'aller voir un psy) si je vous l'imposais, vous me demanderiez si je vous crois folle, le conseil on ne peut le trouver qu'en soi Chat-Accueil (20:58:31): mais on peut avoir besoin des autres pour y voir clair 
Chat-Accueil (21:02:06): la façon dont nous (h ou f) réagirions n'a vraiment aucune importance ou valeur, car nous ne sommes pas vous et vous êtes la seule à "sentir", découvrir, imaginer ce qui peut être bien pour vous et en cela on peut vous aider à y voir clair car ce qui est bon pour vous ne l'est pas nécessairement pour moi (cela vaut au moral comme au physique (si vous ne pouvez pas manger de chocolat et moi oui... ni l'un ni l'autre ne fait mal mais nous devons tous deux faire ce que veut notre corps).

8. Ces explorations ont été réalisées par le Trameur, conçu par Serge Fleury, (EA2290 SYLED/ CLA2T) de l'Université Paris 3 Sorbonne Nouvelle.

9. Groupement 1 : Primo appelant et poly-appelant 1

Groupement 2 : Poly appelant 2, 3, 4, 5

Groupement 3 : Poly appelant 6

Groupement 4 : poly appelant 7

10. Cf. concordancier de ce segment en annexes p. 19.

11. Groupement 1 : Primo appelant et poly-appelant $1,2,4$ et 6

Groupement 2 : Poly appelant 3

Groupement 3 : Poly appelant 5

12. La textualité est ce qui fait d'un texte une suite linguistique signifiante "un ensemble cohérent progressant vers une fin et parvenant à constituer une complétude de sens » (Détrie, Siblot, Verine 2001 : 349).

\section{RÉSUMÉS}

À partir de conversations sous forme de tchats qui émanent d'une association qui lutte contre le suicide, nous nous intéresserons plus particulièrement aux conversations dont les appelants reviennent sur la plateforme (que nous nommerons poly appelants). Reprennent-ils leurs dires là où la conversation antérieure s'est arrêtée? Quels sont les éléments nouveaux dans le récit des participants ? Comment le récit des appelants progresse-t-il au fil des (re)connections? quel(s) versant(s) de l'histoire personnelle est-il, sont-ils mobilisé(s)?

From conversations in the form of chats that emanate from an association that combats suicide, we will focus in particular on the conversations whose callers return to the platform (which we will call poly callers). Do they pick up where they left off in the previous conversation? What are the new elements in the participants' story? How does the appellants' story progress over the (re) connections? what side of personal history is used?

\section{INDEX}

Mots-clés : Discours, conversation, énonciation, dialogisme

Keywords : Discurse, conversation, enunciation, dialogism 
AUTEUR

FRÉDÉRIC PUGNIÈRE-SAAVEDRA

Université Bretagne Sud 